\title{
Final Technical Report
}

Strategic Design and Optimization of Inorganic Sorbents for Cesium, Strontium and Actinides

Project Number DE-FG07-01ER63300

September 2004

Investigators (TAMU TEAM)

Prof. Abraham Clearfield

Dr. Akhilesh Tripathi

Mr. Dmitri G. Medvedev

Mr. Jose Delgado

Mr. Steve Kerlegon

Department of Chemistry

Texas A\&M University

P.O. Box 30012

College Station, TX, 77842-3012

Phone 979-845-2936

Fax 979-845-2370

Clearfield@mail.chem.tamu.edu 


\section{Research Objective:}

The basic science goal in this project identifies structure/affinity relationships for selected radionuclides and existing sorbents. The task will apply this knowledge to the design and synthesis of new sorbents that will exhibit increased cesium, strontium and actinide removal. The target problem focuses on the treatment of high-level nuclear wastes. The general approach can likewise be applied to non-radioactive separations.

\section{Research Progress}

During the third year of the project in addition to continuing our focus along two paths: (1) identifying the structure/affinity relationships for crystalline silicotitanate (CST) and (2) synthesis of new sorbents that include derivatives of CST, a titanosilicate analog of the mineral pharmacosiderite, sodium titanates, we are also conducting (3) insitu crystal growth and ion exchange studies at the X7B beamline of the National Synchrotron Light Source (NSLS), Brookhaven National Laboratory (BNL), Upton, NY, USA for collecting dynamic X-ray powder diffraction data. Based on the results of these studies a pathway for CST crystallization has been proposed.

CST serves as the baseline or alternative technology sorbent for the separation of

${ }^{137} \mathrm{Cs}$ from waste solutions at several of the DOE sites. This material also exhibits a fairly high affinity for strontium, but rather low affinity for plutonium and other actinides. A sodium titanate material, monosodium titanate (MST), serves as the baseline material at the Savannah River Site for strontium and actinide separations from HLW solutions. Recent work demonstrated that pharmacosiderite and sodium nonatitanate (SNT) materials have strontium and actinide removal characteristics similar to that of MST.

This year project members at Texas A\&M completed two detailed structural characterizations and studied in situ crystallization process of CST. Structural studies include CST and niobium-substituted CST (Nb-CST) sorbents before and after exchange 
with strontium and another closely related titanosilicate material with the mineral pharmacosiderite topology (TSP). A complete or partial substitution of elements Ge or

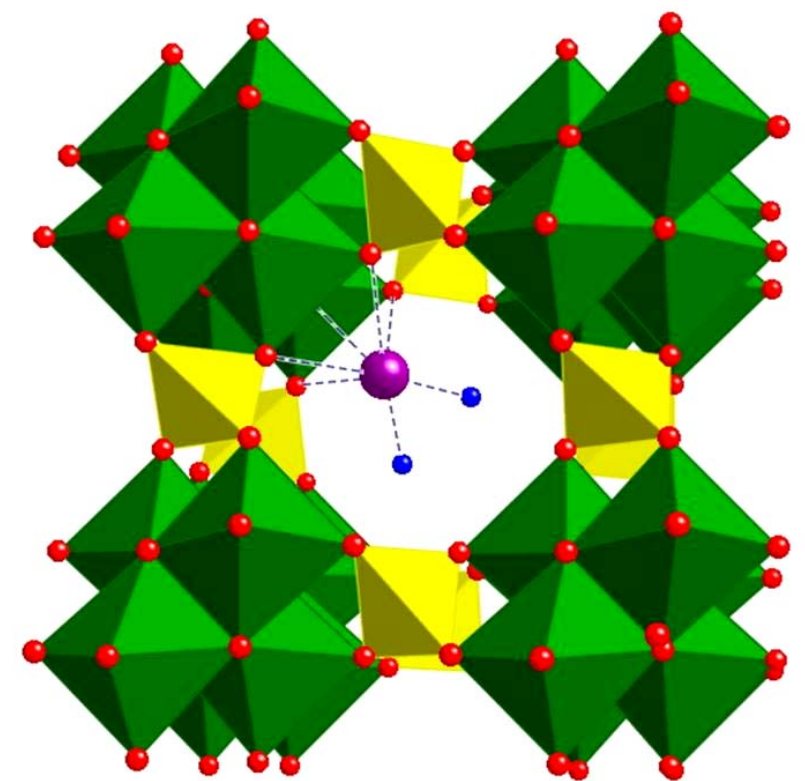

Figure 1. A polyhedral representation (with green $\mathrm{TiO}_{6}$ octahedra and yellow $\mathrm{SiO}_{4}$ tetrahedra) of Nb-CST along [001], strontium (purple spheres) and water (blue spheres) were located in the 8atom ring tunnels. In the CST instead of seven a ten coordinated strontium was observed.

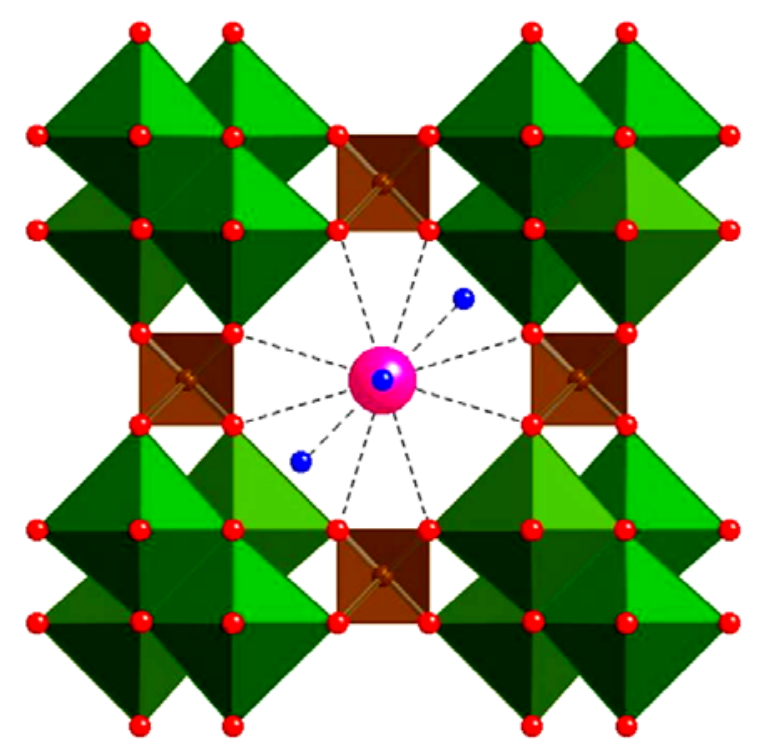

Figure 2. A polyhedral perspective representation of TSP along the [001] plane. Water (blue spheres) and cesium (pink spheres) are located in the 8-atom ring tunnels. $\mathrm{TiO}_{6}$ octahedra are shown in green and $\mathrm{SiO}_{4}$ tetrahedra in brown. The coordination of Cs was found to be directly correlated to the observed exchange capacity.

$\mathrm{Nb}$ was carried out in the framework and the consequent change in the ion exchange properties towards $\mathrm{Cs}$ were studied for $\mathrm{Ti} / \mathrm{Ge} / \mathrm{Si}, \mathrm{Ti} / \mathrm{Si}, \mathrm{Nb} / \mathrm{Ti} / \mathrm{Si}$ and pure $\mathrm{Ge}$ phases. As reported in the previous report also, this research found that the appropriate modification can be made in the structure of CST and TSP materials so as to tune them for higher selectivity for cesium and strontium, as per the requirement. The differences in the uptake of cesium and strontium result from the different coordination environments of these ions in tunnels, that result from various hydration sites in the tunnel (see Figures 1 and 2). The origin of selectivity appears to arise from the higher coordination number of cesium or strontium and the particular site occupied by them in the tunnel. 
The exchange of $\mathrm{Sr}^{2+}$ into the proton form of the ion exchanger CST, $\mathrm{Na}_{2} \mathrm{Ti}_{2} \mathrm{O}_{3}\left(\mathrm{SiO}_{4}\right) \cdot 2 \mathrm{H}_{2} \mathrm{O}$ was examined in FY04 by means of X-ray powder diffractometry. At the $25 \% \mathrm{Sr}^{2+}$ exchange level, the strontium ion was found near an oxo-framework oxygen midway between two adjacent faces of the tetragonal unit cell. It is bonded to five framework oxygen atoms and five water molecules (Figure 3). The $\mathrm{Sr}$ coordination number $(\mathrm{CN})$ is reduced to 9 in a CST phase for which $16 \mathrm{~mol} \%$ of the $\mathrm{Ti}$ was replaced by $\mathrm{Nb}$ (V). It is previously shown that at a higher level

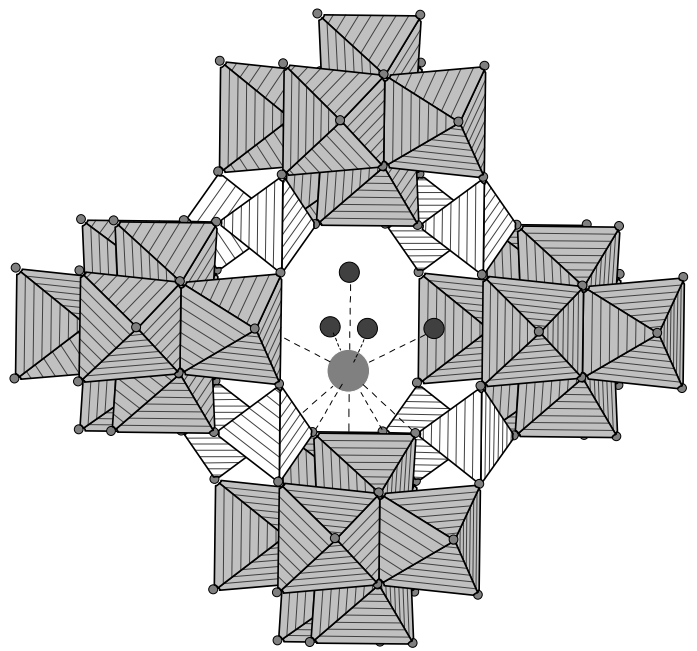

Figure 3. A perspective view of the polyhedral representation in $25 \% \mathrm{Sr}-\mathrm{TS}$. The eight ring tunnel along $c$-axis depicts a ten coordinate $\mathrm{Sr}$ complex. Sr1 is coordinated by five framework oxygen atoms and five water molecules. of $\mathrm{Nb}$ substitution $(25 \mathrm{~mol} \%$ ) the $\mathrm{CN}$ is further reduced to seven. This change in $\mathrm{CN}$ is opposite to what is observed for $\mathrm{Cs}^{+}$where the $\mathrm{CN}$ is eight for the Ti phase and twelve for the $\mathrm{Ti}_{1.5} \mathrm{Nb}_{0.5}$ phase. The changes in $\mathrm{CN}$ are accompanied by a series of changes in the space group to accommodate occupancy of the exchange sites by the cations and water molecules. The selectivity of the exchanger is governed by the strength and number of bonds formed by the exchange reactions. In this study we have shown that the CST framework was able to accommodate four different space groups in two different crystal systems. The original sodium phase belongs to space group $\mathrm{P} 4_{2} / \mathrm{mcm}$. On exchanging the $\mathrm{Na}^{+}$for protons the space group shifts to $\mathrm{P} 4_{2} / \mathrm{mbc}$ in which the $\mathrm{ab}$ diagonals becomes the new $\mathrm{A}$ and $\mathrm{B}$ axes. This

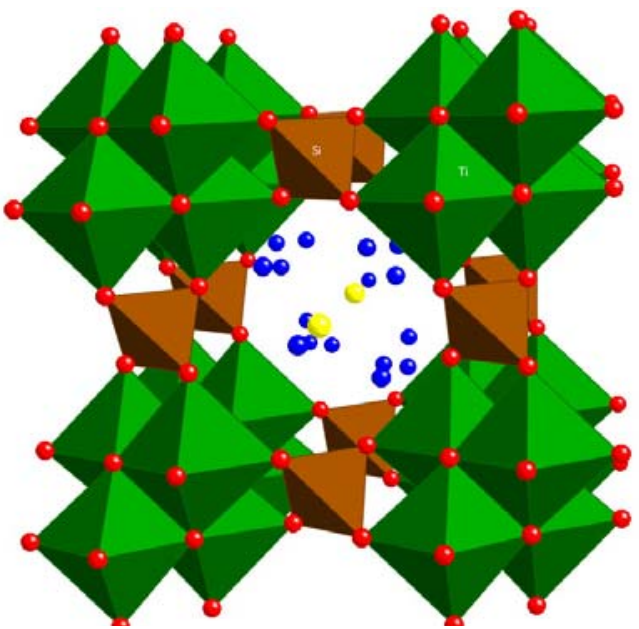

Figure 4. A polyhedral perspective representation of TSP along the [001] plane. Water (blue spheres) and $\mathrm{Na}$ (yellow spheres) are located in the 8-atom ring tunnels. structural change arises because the protons bond to the oxygen atoms that form the $\mathrm{Ti}_{4} \mathrm{O}_{4}$ cubane-like cluster and the water content of the unit cell is $6 \mathrm{H}_{2} \mathrm{O}$ or 1.5 per two Ti. The covalently bonded protons and water molecules 
form a highly symmetrical hydrogen bonded array with the framework oxygen atoms. As $\mathrm{Sr}^{2+}$ replaces $25 \%$ of the protons, the water molecules rearrange themselves into three different sites requiring a change in space group to $\mathrm{Cmmm}$ to properly site the $\mathrm{Sr}$ along the larger number of different water sites. This trend continues as the $\mathrm{Sr}$ content doubles, the strontium occupies two different sites with two different arrangements of the water molecules in the two metal coordination spheres. Interestingly the $16 \% \mathrm{Nb}$ containing phase returns to the original space group of the $\mathrm{Na}-\mathrm{CST}$ compound and the $25 \% \mathrm{Nb}$ phase at a loading of only 0.2 mols of Sr. However this latter phase also contained the framework $\mathrm{Na}^{+}$.

Several new phases synthesized at Texas A\&M during FY04 continued to focus on a titanosilicate (TSP) material with a pharmacosiderite type structure. The pharmacosiderite structure has a similar tunnel structure to that CST. Unlike CST, which has a 2-dimensional tunnel structure, the cubic structure of this material requires that the tunnels in which ion exchange occurs are 3-dimensional (Figure 2 and 4).

\section{A 3-dimensional}

tunnel structure opens
the material more,
which should increase
the effective number of
exchange sites and
facilitate mass transfer

to the ion-exchange sites. We synthesized a range of compositions with pharmacosiderite topology and studied their ion exchange properties. The origin of selectivity for Figure. 5. $\mathrm{K}_{\mathrm{d}}$ values for CsH-TGSG-P5, CsH-TGSG-P6, CsH-TGSG$\mathrm{P7}, \mathrm{CsH}-\mathrm{TGG}-\mathrm{P} 8$ as a function of unit cell dimensions. The unit cell dimension increase from phase P5 to P8. Simulant composition: $0.05 \mathrm{M}$ $\mathrm{NaOH}, 0.05 \mathrm{M} \mathrm{NaNO}_{3},{ }^{137} \mathrm{Cs}$-trace.

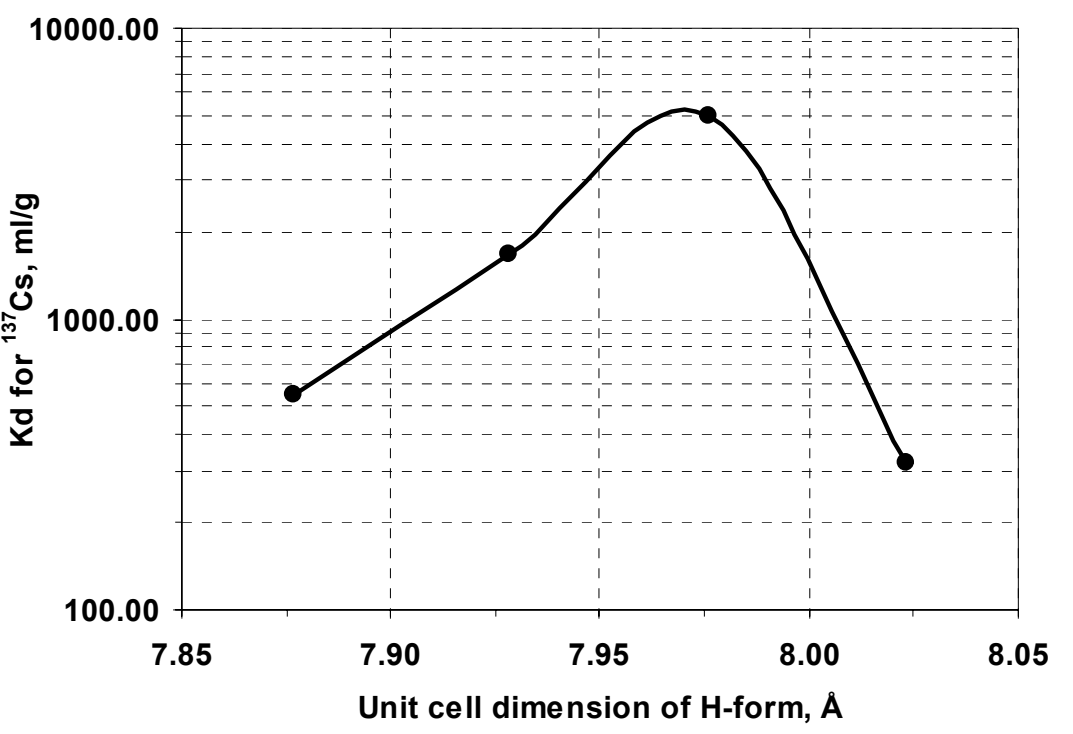

$\mathrm{Cs}^{+}$cations in these phases has 
been correlated with the atomic structure. In the first set of mixed $\mathrm{Ti} / \mathrm{Ge} / \mathrm{Si}$ phases, the size of the eight ring was systematically increased in order to shift the Cs cation as close as possible to the ideal site $(1 / 2,1 / 2,0)$ that is usually occupied by the smaller $\mathrm{K}$ cations. This Cs movement in turn shifts the position of water molecules (Ow1) allowing enough space in the center of the channel $(1 / 2,1 / 2,1 / 2)$ for another half (in Cs-TGSG-P2 and P4) or fully (in Cs-TGSG-P3) occupied water molecule (Ow2) to move in. The highest $\mathrm{K}_{\mathrm{d}}$ values were observed in the phase that has Cs ion closest to the ideal site (Figure 5) which results in the highest coordination number resulting from an additional water molecule at $(1 / 2,1 / 2,1 / 2)$. In Cs-TGG-P1, Cs ion farthest from the ideal site and no additional water molecule was observed at $(1 / 2,1 / 2,1 / 2)$. This site is too close to the fully occupied site with Ow1. In Nb-substituted TSP phase, better $\mathrm{K}_{d}$ values can be correlated to the decrease in charge balance requirement in the channels resulting from the substitution of $\mathrm{Nb}^{\mathrm{v}}$ for $\mathrm{Ti}^{\mathrm{iv}}$. Disorder of the Cs cations was observed in the ideal site $(1 / 2$, $1 / 2,0)$ along with $\mathrm{K}$ besides the usual site $(1 / 2,1 / 2, \mathrm{z})$ in contrast to the non $\mathrm{Nb}$ substituted $\mathrm{Ti} / \mathrm{Si}$ phase. The pure Ge phase appears to be the best $\mathrm{K}$ sorbent as it has shortest K-O distances and does not show ion exchange behavior under ordinary conditions. In general, it is difficult to remove the cations from the K-form of TiSi phase as they 'plug up' well in the ideal site with eight equal bonds with framework oxygen and four equal bonds with water molecules compared to the 'rattling' Na cations in CST.

This year we carried out a series of crystallization experiments both ex- and insitu to study the crystal growth of CST. The parameters that influenced the product composition the most were detailed via ex-situ synthesis studies. The crystallization of the CST was then carried out in-situ using the synchrotron radiation facility at the NSLS, $\mathrm{BNL}$, for collecting dynamic X-ray powder diffraction spectra. Based on the results of these studies a pathway for CST crystallization has been established.

In situ studies represent a unique way to optimize the synthesis toward producing more crystalline materials via understanding of the pathway, kinetics and mechanism of the crystallization process. Knowledge of the pathway of the crystallization process of the CST is essential because it may result in cost cutting modification of the commercial 
crystallization

process as well as

ideas for novel (001) SNT

materials.

Considerable

information on

crystallization can

be obtained in ex-

situ experiments, by

stopping the reaction

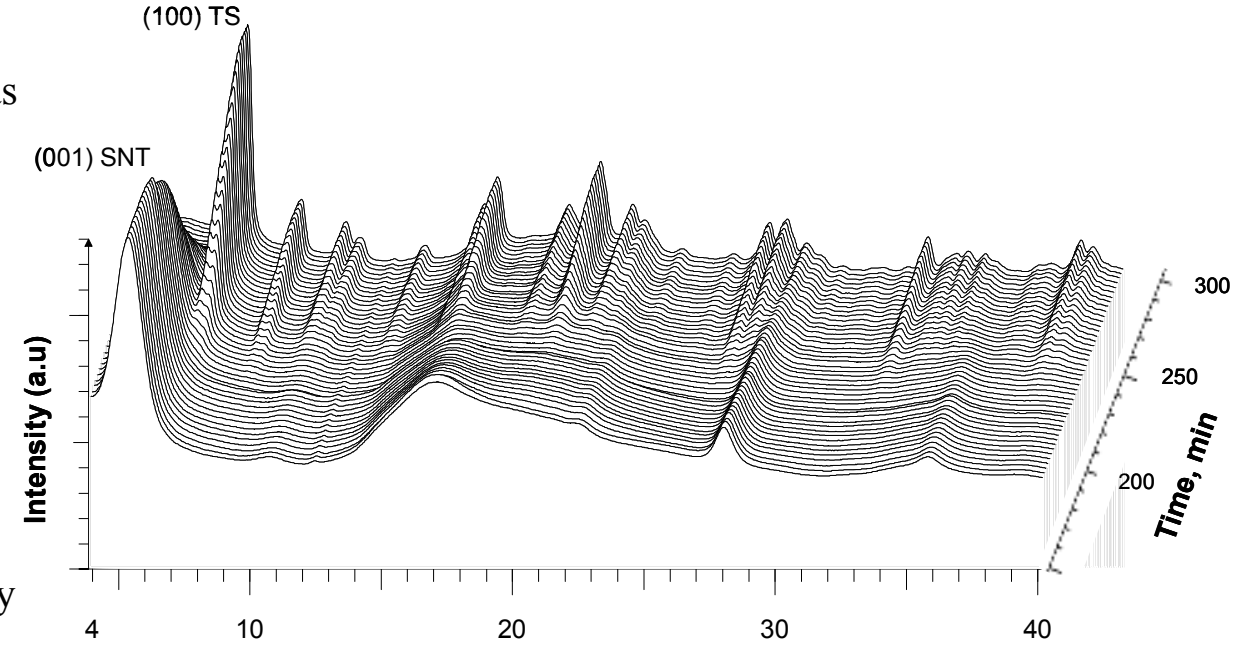

at regular intervals,

or by variation of

$2 \theta$, degrees

Figure 6. Time-resolved X-ray powder diffraction spectra of SNT-TS transformation for the gel with composition: $1.0 \mathrm{TiO}_{2}: 1.98 \mathrm{SiO}_{2}: 6.77 \mathrm{Na}_{2} \mathrm{O}: 218 \mathrm{H}_{2} \mathrm{O}$. Powder patterns are collected in $2.5 \mathrm{~min}$ interval.

synthetic

parameters and collecting X-ray diffraction patterns of the samples. However, this process is generally time consuming, provides limited information, and can be prone to misleading results if in the process of extracting samples the reaction is affected. In-situ $\mathrm{X}$-ray diffraction is a convenient tool to obtain more detailed data about crystal growth of a material, which provides a continuous record of the crystallization process while leaving the reaction undisturbed. It gives easy and rapid information about any intermediate and short-lived phases formed during the hydrothermal reaction. Figure 6 shows a 3D plot of the X-ray diffraction spectra as a function of time during the TS gel heating. As can be seen from the figure the process of CST crystallization starts with the formation of a phase having a broad peak at about 9.5-10 $\AA$. This phase was identified as the SNT phase which is a leading alternative candidate for Sr removal. The phase starts forming at an early stage of reaction, which is confirmed by collecting of X-ray diffraction powder pattern of the dried starting gel. The intensity of the (001) SNT reflection does not change as the reaction progresses until the growth of the CST phase begins. The process of transformation of the SNT phase to the CST phase started after 1 hour of constant heating at $220^{\circ} \mathrm{C}$, with rapid intensity decrease of SNT peaks and ingrowth of CST peaks. The whole process of transformation lasted about 45 minutes. During the period following the transformation to TS, no significant changes occurred, 
except a slight increase in CST peak intensities. No other phases were observed over the course of experiment. As evident from the in-situ TS crystallization experiment, the strontium selective SNT phase precedes the crystallization of the cesium selective TS phase. Thus a mixture of the phases can be obtained if the reaction is stopped during the transformation process, yielding a material which is selective both for Cs and Sr. In order to synthesize a SNT-CST mixed phase several syntheses were carried out ex-situ in 100 $\mathrm{ml}$ pressure vessels by stopping the reaction after different periods of heating at $200^{\circ} \mathrm{C}$. The SNT phase formed after 1 hour of heating while in 10 hours, the TS phase started to crystallize. In 24 hours, the SNT phase completely converted to the TS phase. Several mixtures of phases were obtained between $9-11$ hours of heating at $200^{\circ} \mathrm{C}$, with different ratios of two phases.

The main result of this study is the discovery of the route of TS crystallization. The fact that the SNT precedes the formation of TS phase is of particular interest because SNT itself has the ability to remove strontium and actinides from highly alkaline media. In fact, National Research Council in its report referred to the utilization of SNT for strontium and actinides removal as "an appealing alternative from the standpoint of reliable sources" and stated that "... a range of possibilities can be considered - for example SNT could be used in an ion-exchange mode, either alone or in some combination with crystalline silicotitanate". Being used for cesium removal, the TS phase when utilized in combination with SNT would not only remove cesium, but also strontium and actinides from liquid HLW. Besides, the mixtures of SNT and TS phases in different ratios can be synthesized from one precursor by adjusting the time of the hydrothermal treatment. The synthesis of both materials from one precursor would reduce the manufacturing costs dramatically.

During FY04, SRTC team members evaluated several new materials prepared by the Sandia and Texas A\&M team members for cesium, strontium and actinide removal performance with simulated and actual tank waste solutions. Materials tested included sodium nonatitanate in $\mathrm{H}$-form, niobium-substituted sodium nonatitanate, substituted TSP pharmacosiderites and SNT-CST mixtures. The results indicated that several of these materials exhibited a higher affinity for plutonium relative to strontium than the baseline 
material used at the Savannah River Site. This SNT-CST mixture phase exhibited slower strontium removal kinetics compared to the other pure SNT and MST samples. Plutonium and neptunium removal characteristics were similar to those of the other SNT materials, which is remarkable. Previous testing with commercially prepared CST materials showed that this sorbent exhibits very little affinity for actinides. Given the reduced SNT content in the SNT-CST material, the relatively good plutonium and neptunium removal performance reflects either increased actinide removal characteristics of the SNT or the CST phase.

Overall, the performance of the H-SNT and the SNT-CST samples showed evidence of enhanced affinities for strontium and actinides and increased strontium removal kinetics for the H-SNT sample (Figure 7-9). We believe that the SNT-CST mixed phase material exhibits promise for one-step treatment process to remove cesium, strontium and actinides from HLW solutions. We plan to pursue further development of this material.

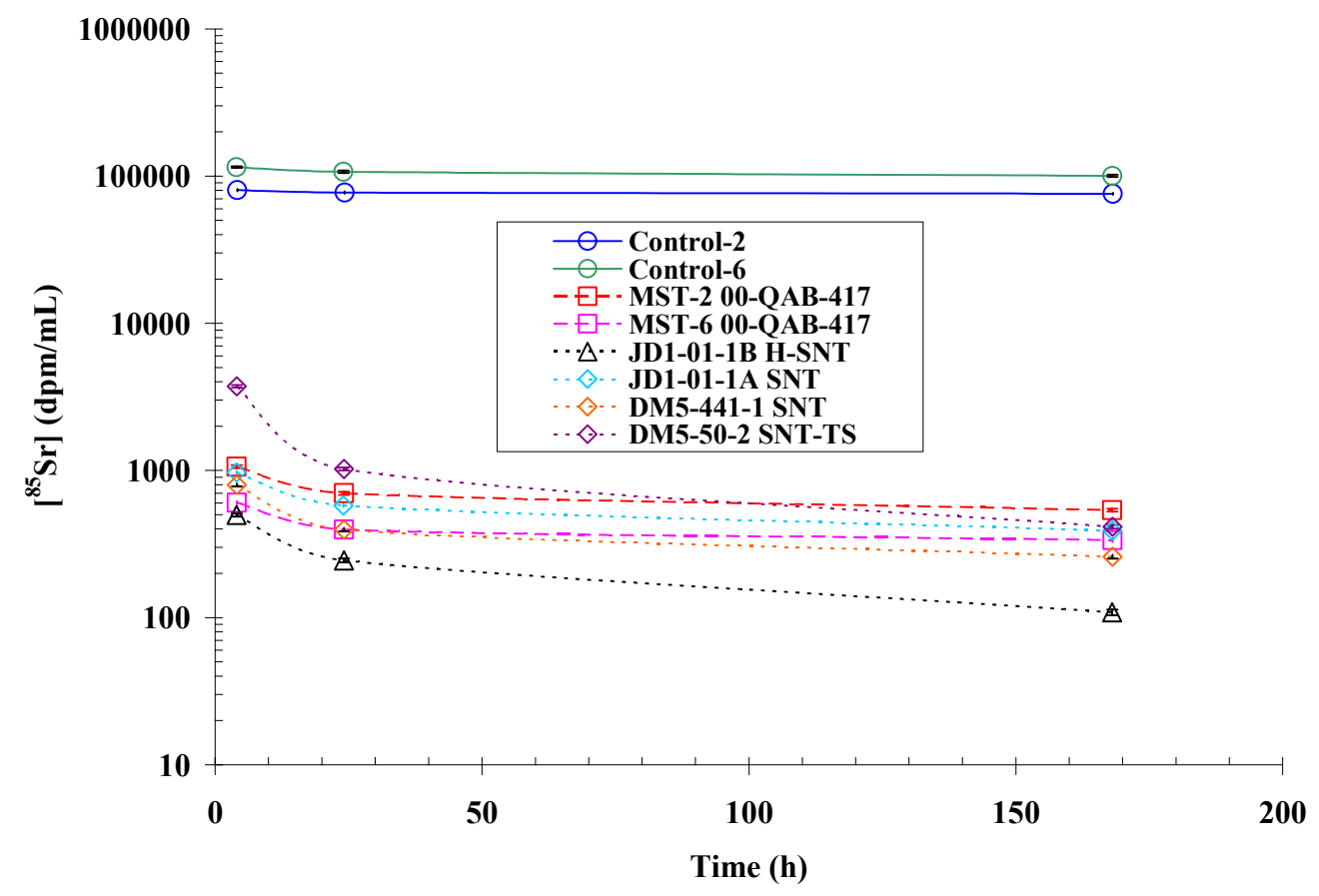


Figure 6. Plot of ${ }^{85} \mathrm{Sr}$ activity versus time upon contact of TAMU prepared SNT and SNT-TS materials with simulated SRS waste solution (courtesy D. T. Hobbs, Savannah River National Laboratory).

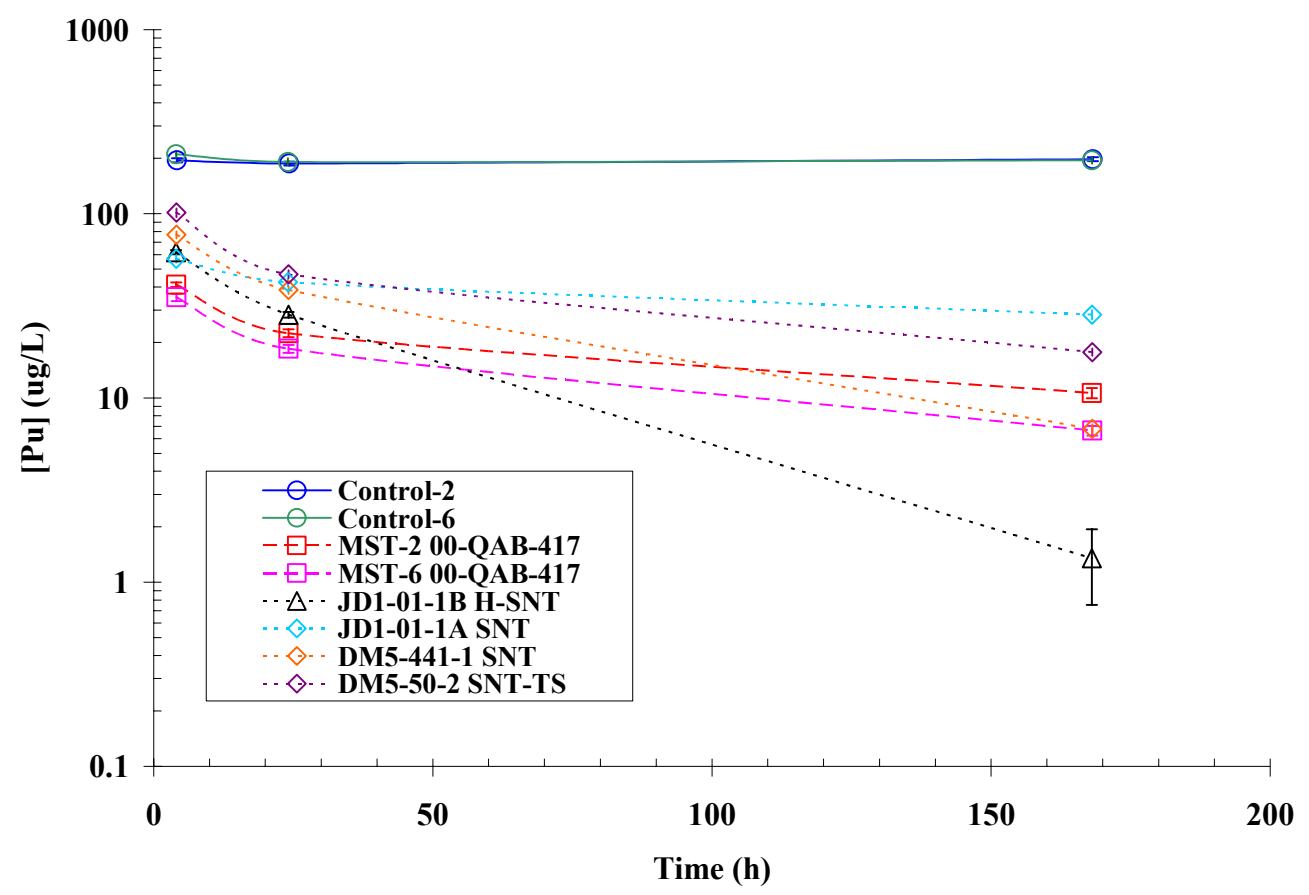

Figure 7 Plot of plutonium concentration versus time upon contact of TAMU prepared SNT and SNT-TS materials with simulated SRS waste solution (courtesy D. T. Hobbs, Savannah River National Laboratory) 


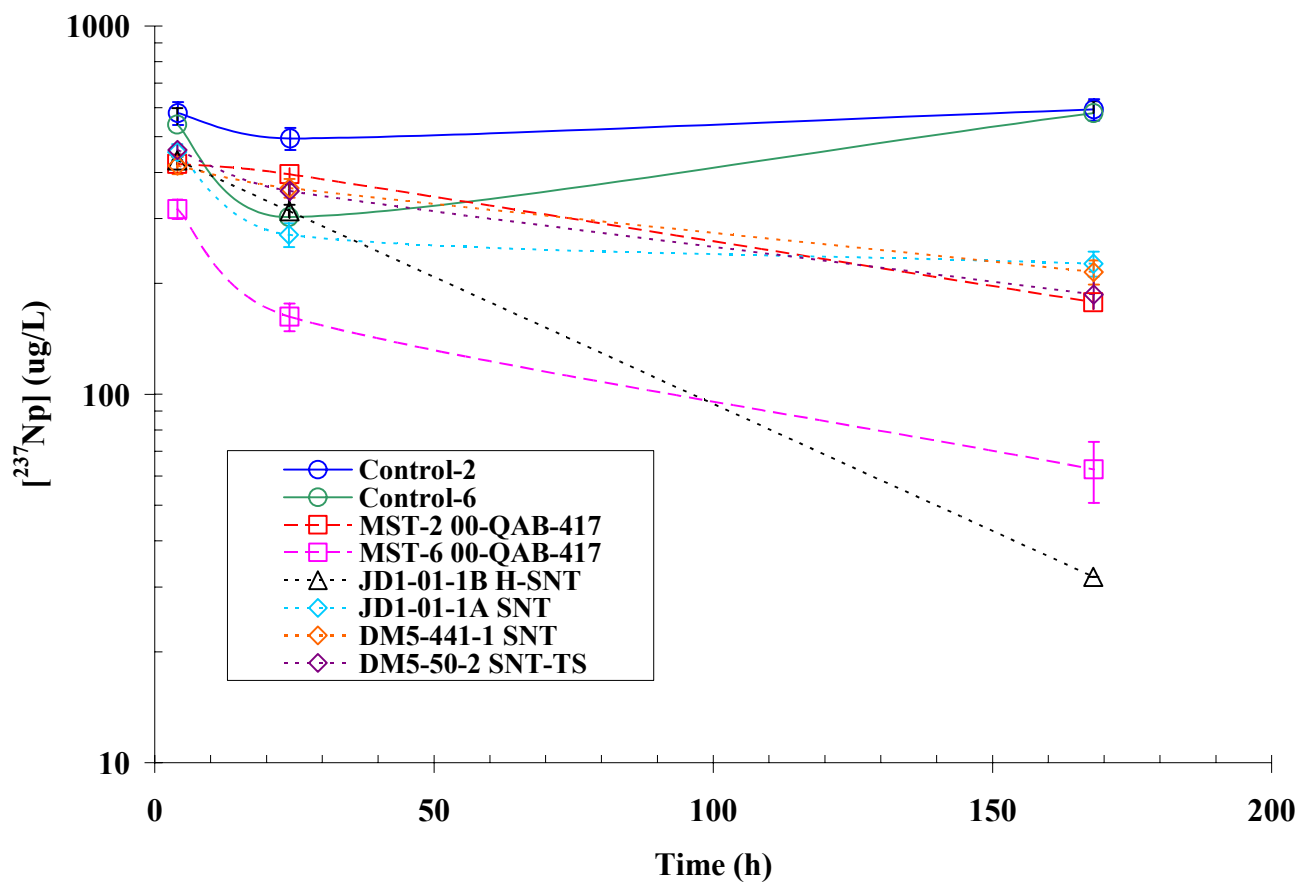

Figure 8. Plot of ${ }^{237} \mathrm{~Np}$ concentration versus time upon contact of TAMU prepared SNT and SNT-TS materials with simulated SRS waste solution (courtesy D. T. Hobbs, Savannah River National Laboratory).

The progress made in new materials development under this EMSP project resulted in producing optimized CST (Nb-CST), development of heteroatom substituted pharmacosiderites (TSP) and in-situ routes for optimizing synthesis and ion exchange in these materials. The mixed phase sample, SNT-TS, represents a novel material comprised of two sorbent materials, SNT and a titanosilicate with the structure of the crystalline silicotitanate ion exchange material. Further development of these materials is in progress and we are awaiting further funding. Dr. D. Hobbs of SRTC serves as the principal investigator for this project with M. Nyman of SNL and A. Clearfield of Texas A\&M University (TAMU) serving as the leads for synthesis of optimized materials. Other team members in TAMU are, Post Doctoral research associate Akhilesh Tripathi, graduate student Dmitri Medvedev and undergraduate Steve Kerlegon. 


\section{Planned Activities}

Modeling activities will extend to $\mathrm{Sr}^{2+}$ exchanged germanium substituted pharmacosiderites as well as to the development of minimum energy models for other pharmacosiderites. Since Sr exchange pharmacosiderites results in mixture of phases we plan to prepare single crystals of Ge form of pharmacosiderites. In situ work at NSLS will include Sr exchange studies in CST and TSPs and crystal growth conditions for mixed niobium substituted SNT-CST phase. Work has already been initiated in this direction.

\section{$\underline{\text { Publications and Presentations }}$}

Peer reviewed publications of work completed in this project include the following:

1. "A Molecular Modeling Investigation of Cation and Water Sitting in Crystalline Silicotitanates" James P. Larentzos, Abraham Clearfield, Akhilesh Tripathi, Edward J. Maginn, Submitted to Journal of Physical Chemistry, 2004.

2. "The Crystal Structures of Strontium Exchanged Sodium Titanosilicates in Relation to Selectivity for Nuclear Waste Treatment" Akhilesh Tripathi, Dmitri G. Medvedev, Abraham Clearfield, In Press: Journal of Solid State Chemistry, 2004.

3. "Optimization of the synthesis of sodium titanium silicate with sitinakite topology: crystallization from sodium nonatitanate phase" Dmitri G. Medvedev, Akhilesh Tripathi, Aaron J. Celestian, John B. Parise, John Hanson, Abraham Clearfield, In Press: Chemistry of Materials, 2004.

4. "Optimizing Cs-exchange in Titanosilicate with the Mineral Pharmacosiderite Topology: Framework Substitution of $\mathrm{Nb}$ and Ge" Akhilesh Tripathi, Dmitri G. Medvedev, Jose Delgado, Abraham Clearfield, Journal of Solid State Chemistry, 177 (8), 2904-2916 (2004).

5. "Selectivity for $\mathrm{Cs}$ and $\mathrm{Sr}$ in $\mathrm{Nb}$-substituted titanosilicate with sitinakite topology" Akhilesh Tripathi, Dmitri G. Medvedev, May Nyman, Abraham Clearfield, Journal of Solid State Chemistry 175(1), 72-83 (2003).

6. "The First Framework Solid Composed of Vanadosilicate Clusters" Akhilesh Tripathi, Timothy Hughbanks, Abraham Clearfield, Journal of the American Chemical Society, 125(35), 10528-10529 (2003). 
Presentations of work completed in this project at technical conferences and meetings include the following:

1. Presented lecture on Porous materials, Chemistry Department, University of San Juan, Puerto Rico, May 21, 2004.

2. "Synchrotron time resolved x-ray diffraction of Cs exchange into molecular sieve HCST" Aaron J. Celestian, Dmitri Medvedev, Akhilesh Tripathi, John B. Parise, Abraham Clearfield, Jonathan C. Hanson, Abstracts of Papers, 227th ACS National Meeting, Anaheim, CA, United States, March 28-April 1, 2004.

3. "Time resolved diffraction studies on titanosilicate with mineral sitinakite topology: From crystallization to ion-exchange" Dmitri Medvedev, Akhilesh Tripathi, Abraham Clearfield, Aaron J. Celestian, John B. Parise, Jonathan C. Hanson, Abstracts of Papers, 227th ACS National Meeting, Anaheim, CA, United States, March 28-April $1,2004$.

4. "Optimizing $\mathrm{Cs}$ and $\mathrm{Sr}$ selectivity in titanosilicate with pharmacosiderite topology: framework substitution of $\mathrm{Nb}$ and Ge" Akhilesh Tripathi, Dmitri Medvedev, Jose Delgado, Abraham Clearfield, Abstracts of Papers, 227th ACS National Meeting, Anaheim, CA, United States, March 28-April 1, 2004.

5. "Evaluation of new inorganic sorbents for strontium and actinide removal from highlevel nuclear waste solutions" D.T. Hobbs, May D. Nyman, Abraham Clearfield, Akhilesh Tripathi, Dmitri Medvedev, Abstracts of Papers, 227th ACS National Meeting, Anaheim, CA, United States, March 28-April 1, 2004.

6. "Origin of selectivity in tunnel type inorganic ion exchangers" Abraham Clearfield, Akhilesh Tripathi, Dmitri Medvedev, Jose Delgado, May Nyman, Abstracts of Papers, 227th ACS National Meeting, Anaheim, CA, United States, March 28-April $1,2004$.

7. "Origin of selectivity in tunnel type inorganic ion exchangers" Abraham Clearfield, Akhilesh Tripathi, Dmitri Medvedev, Jose Delgado, May Nyman, Preprints of Extended Abstracts presented at the ACS National Meeting, American Chemical Society, Division of Environmental Chemistry, 44(1), 358-359 (2004).

8. Presented paper at DOE symposium on Origin of Selectivity in Tunnel Type Inorganic Ion Exchangers with A. Tripathi, D. Medvedev, J. Delgado and M. Nyman. Five additional papers and posters were presented by students and post-docs, Mar. 2831, 2004.

9. Presented paper at Pittsburgh Diffraction Conference, Rutgers University on History of Structure Solutions from Powder Data at Texas A\&M University, Oct. 30-31, 2003. 
10. "Structure and the origins of ion exchange selectivity in tunnel type titanium silicates" Abraham Clearfield, Akhilesh Tripathi, Dmitri Medvedev, Xiang Ouyang, Abstracts of Papers, 226th ACS National Meeting, New York, NY, United States, September 7-11, 2003.

11. "Investigation of strontium ion-exchange process in titanium silicate" Dmitri Medvedev, Akhilesh Tripathi, Abraham Clearfield, Abstracts of Papers, 226th ACS National Meeting, New York, NY, United States, September 7-11, 2003.

12. "Structural basis of selectivity: Exchange of radioactive cesium and strontium ions in hydrous mixed metal oxides of $\mathrm{Sb}, \mathrm{Nb}, \mathrm{Si}, \mathrm{Ti}$ and $\mathrm{W}$ with the pyrochlore structure" Akhilesh Tripathi, Dmitri Medvedev, Michael Gustavson, Michael Sarahan, Abraham Clearfield, Abstracts of Papers, 226th ACS National Meeting, New York, NY, United States, September 7-11, 2003. 